\title{
Officers and Council Members of the British Society for the History of Science, 1947-97
}

\section{Citation}

Browne, Janet. 1997. Officers and council members of the British Society for the History of Science, 1947-97. The British Journal for the History of Science 30, no. 1: 77-89

\section{Published Version}

http://dx.doi.org/10.1017/S0007087496002919

\section{Permanent link}

http://nrs.harvard.edu/urn-3:HUL.InstRepos:3353946

\section{Terms of Use}

This article was downloaded from Harvard University's DASH repository, and is made available under the terms and conditions applicable to Other Posted Material, as set forth at http:// nrs.harvard.edu/urn-3:HUL.InstRepos:dash.current.terms-of-use\#LAA

\section{Share Your Story}

The Harvard community has made this article openly available.

Please share how this access benefits you. Submit a story.

Accessibility 
BJHS, 1997, 30, 77-89

\title{
Officers and council members of the British Society for the History of Science, 1947-97
}

\author{
JANET BROWNE*
}

As described elsewhere in this issue of $B J H S$, preliminary steps towards founding a society for the history of science in Britain were taken in 1946. A meeting was held at the Science Museum, London, on 22 November 1946, chaired by Herbert Dingle, at which Gavin de Beer formally proposed the foundation of a history of science society, seconded by Michael Roberts. A provisional committee was appointed to draw up rules and a constitution. It included:

Dr H. P. Bayon

Professor G. R. de Beer

Mr C. N. Bromehead

Mr F. C. H. Butler (Secretary)

Mr J. G. Crowther

Professor H. Dingle

Dr S. Lilley

Mr Michael Roberts
Mr H. W. Robinson

Dr H. Shaw

Dr Charles Singer

Mrs Dorothea Singer

Dr F. Sherwood Taylor

Dr H. Hamshaw Thomas

Dr E. Ashworth Underwood

This provisional committee met three times over the next few months.

The British Society for the History of Science came into formal being on Monday, 5 May 1947, in the meeting room of the Royal Society, Burlington House, with Dr Sherwood Taylor in the chair. Elections for a president (Charles Singer), vice-presidents, officers and council members took place (see Table 1) and the new constitution was approved. The business of the meeting included a proposal by J. G. Crowther 'that the Society should have a Journal', seconded by H. P. Bayon, although nothing further was agreed. Seventyone founder members joined at a subscription of one guinea (see the list, p. 79).

The new Society was active during its first year. From the very first council meeting, held on 14 May 1947 in the Science Museum, the question of publications became an issue, and a possible journal, newsletter and 'authoritative translations of classical scientific works' were in turn explored. ${ }^{1}$ A Philosophy of Science Group was set up, run as a relatively independent wing from February 1948 to November 1958 when a separate society was established. This group was initiated by Herbert Dingle at University College London, where most of the early meetings took place. Many eminent philosophers of science became members. The BSHS itself ran to 174 members by 1 June 1948 . That month, the Society's slim, twenty-four page Bulletin began publication under the acting editorship of Samuel Lilley, passing into the hands of N. H. de V. Heathcote in April 1950. The Bulletin included meeting reports, abstracts of papers, presidential addresses, book reviews and

* Wellcome Institute for the History of Medicine, 183 Euston Road, London NW1 2BE.

1 Minutes of the Society, Science Museum Library, BSHS Archive, 1/1. 
Table 1. Foundation officers and council, British Society for the History of Science, 1947-48. Drawn from the Bulletin of the British Society for the History of Science (1949), 1, 3-4

\begin{tabular}{ll}
\hline $\begin{array}{l}\text { President: Dr Charles Singer } \\
\text { Vice-presidents: }\end{array}$ & \\
$\begin{array}{l}\text { Professor H. Dingle } \\
\text { Dr E. J. Holmyard }\end{array}$ & $\begin{array}{l}\text { Professor J. R. Partington } \\
\text { Sir Arthur MacNalty }\end{array}$ \\
Hon. secretary: Mr F. C. H. Butler & Dr F. Sherwood Taylor \\
Hon. treasurer: Mr H. W. Robinson & \\
Council; & \\
Dr H. P. Bayon & \\
Professor G. R. de Beer & Dr D. McKie \\
Mr C. N. Bromehead & Mr H. T. Pledge \\
Mr J. G. Crowther & Dr H. Shaw \\
Dr Dorothy Feyer & Dr H. Hamshaw Thomas \\
Dr S. Lilley & Sir Henry Thomas \\
\end{tabular}

miscellaneous notices. It ran somewhat erratically from 1949 to 1961 (vols. 1 and 2, 258 pp. and $116 \mathrm{pp}$. respectively), towards the end being published as an annex to Annals of Science. It was superseded in 1962 by the British Journal for the History of Science (vol. 1 onwards) under the editorship of H. D. Anthony. The Bulletin also produced occasional supplements on the activities of the Philosophy of Science Group. During the first year the Society held three public meetings in the Science Museum for the reading of scholarly papers and discussion: the papers had to be approved by the Publications sub-committee before being read. Tickets to these meetings were issued to London students but it was thought by council members that students would mostly be too busy to attend.

At the Annual General Meeting on 4 May 1948 Charles Singer initiated the practice of delivering a presidential address. His address, on 'The Role of the History of Science', is reprinted in this issue of the BJHS. Subsequent milestones include the award of Honorary Membership to prominent figures (a practice long since ceased), beginning with George Sarton who received the honour in person on 9 May 1948; the official incorporation of the Society as a company limited by guarantee under the Societies Act on 6 May 1956, and as a Registered Charity on 1 January 1961; the introduction in 1980 of a biennial Singer Prize for an outstanding student essay; and the foundation of an Education Section in 1987. In 1969 W. H. Brock produced the first List of Theses and Dissertions; in 1980 Peter Collins began the Newsletter; in 1992 Geoffrey Cantor published the first BSHS Guide to History of Science Courses in Britain. The Monograph series has gone from strength to strength since its inauguration in 1978. The Society is still represented in the international organizations that were involved in its foundation. 
OFFICERS AND COUNCIL MEMBERS OF THE BRITISH

SOCIETY FOR THE HISTORY OF SCIENCE, 1947-97

Officers are listed chronologically according to first date of office, and council members according to electoral year. The Society's year used to run from May to May.

\section{Presidents}

C. Singer, 1947-49

J. R. Partington, 1949-51

F. Sherwood Taylor, 1951-53

H. Hamshaw Thomas, 1953-55

H. Dingle, 1955-57

E. Ashworth Underwood, 1957-62

T. Martin, 1962-64

A. C. Crombie, 1964-66

A. R. Hall, 1966-68

G. J. Whitrow, 1968-70

W. P. D. Wightman, 1970-72

J. A. Chaldecott, 1972-74

Vice-presidents

H. Dingle, 1947-50, 1953-55, 1957-60, 1961-64

E. J. Holmyard, 1947-50

A. MacNalty, 1947-49

J. R. Partington, 1947-49, 1951-55, 1958-59

Dorothea Singer, 1947-50

F. Sherwood Taylor, 1947-50, 1955-56

C. Singer, 1949-56

H. Hamshaw Thomas, 1949-51, 1955-58, 1959-61

H. Butterfield, 1950-52

D. McKie, 1950-53, 1958-61

C. E. Raven, 1950-52

E. Ashworth Underwood, 1950-53, 1962-64

A. C. Crombie, 1952-55, 1962-64, 1966-68

L. Rosenfeld, 1952-55

R. S. Whipple, 1953-54

A. Armitage, 1955-58, 1961-64

T. Martin, 1955-57, 1958-61, 1964-67
M. P. Crosland, 1974-76

D. W. Waters, 1976-78

W. H. Brock, 1978-80

R. Fox, 1980-82

J. B. Morrell, 1982-84

G. L'E. Turner, 1984-86

C. A. Russell, 1986-88

R. G. W. Anderson, 1988-90

H. S. Torrens, 1990-92

G. N. Cantor, 1992-94

D. M. Knight, 1994-96

J. H. Brooke, 1996-

H. Heywood, 1956-59

G. J. Whitrow, 1955-58, 1959-62

E. G. R. Taylor, 1956-59

T. C. S. Morrison-Scott, 1961-62

Margaret Rowbottom, 1959-62

D. Chilton, 1962-63, 1971-74

F. Greenaway, 1962-63, 1964-65

D. H. Follett, 1963-66

F. H. C. Butler, 1965-68

A. R. Hall, 1965-66, 1968-69, 1976-79

W. P. D. Wightman, 1966-69, 1972-73

W. A. Smeaton, 1968-71

J. A. Chaldecott, 1969-72, 1974-75

W. T. Stearn, 1969-72

M. P. Crosland, 1971-74

J. D. North, 1971-74

D. W. Waters, 1972-75, 1978-81

M. P. Earles, 1974-76

P. M. Rattansi, 1974-77

Mary B. Hesse, 1975-77

H. G. L. Bevan, 1977-80

R. Fox, 1977-78, 1982-83

J. B. Morrell, 1978-81, 1984-85 
G. L'E. Turner, 1979-84, 1986-87

W. H. Brock, 1980-81

C. A. Russell, 1985-86, 1988-89

R. G. W. Anderson, 1987-88, 1990-91

\section{Secretaries}

F. H. C. Butler, 1947-63

J. A. Chaldecott, 1963-69

M. P. Earles, 1969-74

H. G. L. Bevan, 1974-77

W. F. Bynum, 1977-81

\section{Treasurers}

H. W. Robinson, 1947-49

F. A. Freeth, 1949-52

H. R. Calvert, 1952-63

D. Chilton, 1963-69

G. L'E. Turner, 1969-79

\section{Editors}

S. Lilley, 1948-49

N. H. de V. Heathcote, 1948-61

H. D. Anthony, 1961-65

M. P. Crosland, 1965-71

R. Fox, 1971-77

\section{Assistant editor}

Mary B. Hesse, 1957-58

Publications secretaries

F. H. C. Butler, 1963-65

Programme secretaries

M. P. Earles, 1965-67

H. G. L. Bevan, 1967-74

A. M. Duncan, 1974-81

B. J. Norton, 1981-85

Newsletter editors

P. M. D. Collins, 1981-85

Mari E. W. Williams, 1985-89
H. S. Torrens, 1989-90, 1992-93

G. N. Cantor, 1991-92, 1994-95

D. M. Knight, 1993-94, 1996-97

J. H. Brooke, 1995-96

G. N. Cantor, 1981-87

J. V. Golinski, 1987-89

C. W. Smith, 1989-95

J. Hughes, 1995-

J. H. Brooke, 1979-85

P. M. D. Collins, 1985-89

R. C. Olby, 1989-93

Mari E. W. Williams, 1993-

N. W. Fisher, 1976-82

D. M. Knight, 1981-88

J. H. Brooke, 1988-93

Janet Browne, 1993-
J. D. North, 1965-71

J. V. Pickstone, 1986-91

S. Pumfrey, 1991-96

J. E. Agar, 1996-

F. A. J. L. James, 1989- 
Monograph series editors

R. Smith, 1981-85

P. J. Weindling, 1990-96

J. G. Smith, 1985-90

J. F. M. Clark, 1996-

External affairs officers

Patricia Fara, 1993-95

D. E. H. Edgerton, 1996-

\section{Membership secretary}

Sally M. Horrocks, 1996-

Council, 1947-48

H. P. Bayon

D. McKie

G. R. de Beer

C. N. Bromehead

H. T. Pledge

J. G. Crowther

H. Shaw

Dorothy Feyer

S. Lilley

H. Hamshaw Thomas

H. Thomas

E. Ashworth Underwood

Council, 1948-49

H. P. Bayon

C. N. Bromehead

F. A. Freeth

A. C. Crombie

S. Lilley

L. Rosenfeld

J. G. Crowther

H. Shaw

V. A. Eyles

H. Hamshaw Thomas

Dorothy Feyer

E. Ashworth Underwood

Council, 1949-50

H. P. Bayon

T. Martin

H. Butterfield

S. F. Mason

A. C. Crombie

D. McKie

V. A. Eyles

L. Rosenfeld

Dorothy Feyer

H. Shaw

S. Lilley

E. Ashworth Underwood

\section{Council, 1950-51}

H. P. Bayon

T. Martin

A. C. Crombie

S. F. Mason

H. Dingle

J. Needham

V. A. Eyles

F. J. North

Dorothy Feyer

L. Rosenfeld

N. H. de V. Heathcote

R. S. Whipple 
Council, 1951-52
A. Armitage
T. Martin
R. S. Clay
J. Needham
A. C. Crombie
F. J. North
C. H. Desch
L. Rosenfeld
H. Dingle
H. Hamshaw Thomas
N. H. de V. Heathcote
R. S. Whipple

Council, 1952-53
A. Armitage
H. Heywood
R. S. Clay
T. Martin
H. Dingle
F. A. Freeth
F. J. North
C. Singer
A. R. Hall
H. Hamshaw Thomas
N. H. de V. Heathcote
R. S. Whipple
Council, 1953-54
A. Armitage
J. A. Chaldecott
R. S. Clay
A. R. Hall
N. H. de V. Heathcote
H. Heywood
E. J. Holmyard
T. Martin
Eva G. R. Taylor
F. Sherwood Taylor
E. Ashworth Underwood
G. J. Whitrow

Council, 1954-55
A. Armitage
J. A. Chaldecott
R. S. Clay
A. R. Hall
N. H. de V. Heathcote
H. Heywood

E. J. Holmyard

T. Martin

Eva G. R. Taylor

F. Sherwood Taylor

E. Ashworth Underwood

Council, 1955-56

J. A. Chaldecott

A. C. Crombie

W. Mays

Joan M. Eyles

Dorothy Feyer

A. R. Hall

H. Heywood

J. R. Partington

D. J. Price

L. Rosenfeld

Eva G. R. Taylor

Council, 1956-57

A. H. G. Alston

J. A. Chaldecott

Joan M. Eyles

Dorothy Feyer

A. C. Crombie

J. R. Partington 
D. J. Price

L. Rosenfeld

Margaret Rowbottom

Council, 1957-58

J. A. Chaldecott

D. Chilton

A. C. Crombie

Dorothy Feyer

Mary B. Hesse

T. Martin

Council, 1958-59

A. Armitage

J. A. Chaldecott

D. Chilton

Dorothy Feyer

F. Greenaway

Mary B. Hesse

Council, 1959-60

A. Armitage

G. Burniston Brown

D. Chilton

Dorothy Feyer

J. S. L. Gilmour

F. Greenaway

Council, 1960-61

H. D. Anthony

A. Armitage

G. Burniston Brown

J. A. Chaldecott

D. Chilton

H. Dingle

\section{Council, 1961-62}

G. Burniston Brown

J. A. Chaldecott

D. Chilton

A. C. Crombie

D. H. Follett

J. S. L. Gilmour
J. F. Scott

C. Singer

J. O. Wisdom

J. R. Partington

L. Rosenfeld

Margaret Rowbottom

J. F. Scott

L. L. Whyte

J. S. Wilkie

Margaret Rowbottom

J. F. Scott

H. Hamshaw Thomas

G. J. Whitrow

L. L. Whyte

J. S. Wilkie

Mary B. Hesse

T. C. S. Morrison-Scott

J. F. Scott

L. L. Whyte

J. S. Wilkie

J. S. L. Gilmour

F. Greenaway

T. C. S. Morrison-Scott

W. A. Smeaton

W. P. D. Wightman

J. S. Wilkie

S. C. Goddard

F. Greenaway

N. H. de V. Heathcote

M. A. Hoskin

T. Martin

W. P. D. Wightman 
Council, 1962-63

G. Burniston Brown

M. A. Hoskin

J. A. Chaldecott

D. H. Follett

J. R. Ravetz

Margaret Rowbottom

S. C. Goddard

W. T. Stearn

N. H. de V. Heathcote

G. J. Whitrow

Mary B. Hesse

W. P. D. Wightman

Council, 1963-64

H. R. Calvert

Dorothy Feyer

S. C. Goddard

N. H. de V. Heathcote

Mary B. Hesse

M. A. Hoskin

D. McKie

J. R. Ravetz

Margaret Rowbottom

W. A. Smeaton

W. T. Stearn

G. J. Whitrow

Council, 1964-65

H. R. Calvert

A. Clow

Dorothy Feyer

A. R. Hall

N. H. de V. Heathcote

M. A. Hoskin

J. N. F. Morris

Margaret Rowbottom

W. A. Smeaton

W. T. Stearn

E. Ashworth Underwood

J. S. Wilkie

Council, 1965-66

H. R. Calvert

D. S. L. Cardwell

J. N. F. Morris

Margaret Rowbottom

E. Clarke

A. Clow

Dorothy Feyer

W. A. Smeaton

W. T. Stearn

F. Greenaway

E. Ashworth Underwood

Council, 1966-67

D. S. L. Cardwell

F. Greenaway

E. Clarke

J. W. Herivel

A. Clow

M. A. Hoskin

W. S. C. Copeman

D. M. Knight

A. M. Duncan

J. N. F. Morris

Dorothy Feyer

W. A. Smeaton

Council, 1967-68

D. S. L. Cardwell

A. M. Duncan

E. Clarke

E. G. Forbes

W. S. C. Copeman

F. Greenaway 
J. W. Herivel

M. A. Hoskin

D. M. Knight

Council, 1968-69

W. H. Brock

A. M. Duncan

E. G. Forbes

J. W. Herivel

M. A. Hoskin

D. M. Knight

L. L. Laudan

Council, 1969-70

W. H. Brock

D. Chilton

A. M. Duncan

E. G. Forbes

J. W. Herivel

D. M. Knight

Council, 1970-71

W. H. Brock

D. Chilton

E. G. Forbes

R. Fox

Marie B. Hall

F. N. L. Poynter

Council, 1971-72

W. H. Brock

R. A. Buchanan

J. K. Crellin

Marie B. Hall

A. G. Molland

P. M. Rattansi

Council, 1972-73

E. J. Aiton

R. A. Buchanan

J. K. Crellin

Marie B. Hall

Mary B. Hesse

A. G. Keller
L. L. Laudan

C. A. Russell

G. L'E. Turner
F. N. L. Poynter

C. A. Russell

W. T. Stearn

G. L'E. Turner

D. W. Waters
F. N. L. Poynter

J. R. Ravetz

C. A. Russell

D. W. Waters

W. P. D. Wightman

R. M. Young

J. R. Ravetz

C. A. Russell

A. I. Sabra

D. W. Waters

G. J. Whitrow

R. M. Young

J. R. Ravetz

J. L. Russell

A. I. Sabra

D. W. Waters

G. J. Whitrow

R. M. Young
A. G. Molland
R. C. Olby
P. M. Rattansi
M. J. S. Rudwick
J. L. Russell
G. J. Whitrow 
Council, 1973-74

E. J. Aiton

W. H. Brock

A. G. Keller

R. A. Buchanan

R. C. Olby

J. K. Crellin

A. R. Hall

Mary B. Hesse

A. D. Orange

P. M. Rattansi

M. J. S. Rudwick

J. L. Russell

Council, 1974-75

E. J. Aiton

W. H. Brock

J. K. Crellin

W. V. Farrar

N. W. Fisher

E. G. Forbes
A. R. Hall
Mary B. Hesse
A. G. Keller
S. F. Mason
A. D. Orange
J. L. Russell

Council, 1975-76

W. F. Bynum

W. V. Farrar

E. G. Forbes

A. R. Hall

S. F. Mason

A. D. Orange

J. R. R. Christie

N. W. Fisher

F. Greenaway

A. G. Keller

J. B. Morrell

R. S. Porter

Council, 1976-77

W. F. Bynum

A. Chapman

J. R. R. Christie

S. F. Mason

R. M. MacLeod

A. J. Meadows

W. V. Farrar

J. B. Morrell

E. G. Forbes

F. Greenaway

R. S. Porter

Gerrylyn K. Roberts

Council, 1977-78

B. Bracegirdle

A. Chapman

J. R. R. Christie

F. Greenaway

A. J. Meadows

J. B. Morrell

R. H. Naylor

J. V. Pickstone

R. S. Porter

Gerrylyn K. Roberts

W. F. Ryan

S. A. Shapin

Council, 1978-79

B. Bracegirdle

A. Chapman

D. J. Bryden

A. J. Meadows

G. N. Cantor

R. H. Naylor 
J. V. Pickstone

Gerrylyn K. Roberts

W. F. Ryan

Council, 1979-80

D. E. Allen

B. Bracegirdle

D. J. Bryden

G. N. Cantor

B. A. Haines

B. J. Norton

Council, 1980-81

D. E. Allen

D. J. Bryden

G. N. Cantor

P. M. D. Collins

J. R. Durant

B. A. Haines

Council, 1981-82

D. E. Allen

R. G. W. Anderson

I. Grattan-Guinness

B. A. Haines

A. R. Hall

Council, 1982-83

R. G. W. Anderson

D. C. Gooding

I. Grattan-Guinness

D. Layton

J. V. Pickstone

Council, 1983-84

R. G. W. Anderson

D. Bloor

Sophie Forgan

D. C. Gooding

I. Grattan-Guinness

Council, 1984-85

D. Bloor

Sophie Forgan
S. A. Shapin
C. W. Smith
R. Smith

J. V. Pickstone

C. A. Russell

W. F. Ryan

S. A. Shapin

C. W. Smith

R. Smith

Ludmilla J. Jordanova

B. J. Norton

C. A. Russell

C. W. Smith

R. Smith

P. B. Wood

Ludmilla J. Jordanova

J. V. Pickstone

C. A. Russell

P. B. Wood

M. Stanley

N. A. Taylor

$\mathrm{H}$. Wallis

P. B. Wood

J. V. Pickstone

M. Stanley

N. A. Taylor

Mari E. W. Williams

D. C. Gooding

M. Hunter 
S. Schaffer

M. Shortland

M. Stanley

Council, 1985-86

J. A. Bennett

D. Bloor

R. Bud

Sophie Forgan

M. Hunter

Council, 1986-87

J. A. Bennett

R. Bud

A. Gabbey

J. C. Henry

M. Hunter

Council, 1987-88

J. A. Bennett

R. Bud

Stella Butler

A. Gabbey

J. C. Henry

Council, 1988-89

Stella Butler

D. E. H. Edgerton

A. Gabbey

J. C. Henry

G. L. Herries-Davies

Council, 1989-90

Janet Browne

Stella Butler

Elspeth Crawford

D. E. H. Edgerton

G. L. Herries-Davies

Council, 1990-91

P. J. Bowler

Janet Browne

Elspeth Crawford

D. E. H. Edgerton

G. L. Herries-Davies
N. A. Taylor

Mari E. W. Williams

R. C. Olby

S. Schaffer

M. Shortland

S. W. Sturdy

R. C. Olby

S. Schaffer

M. Shortland

S. W. Sturdy

R. C. Olby

J. A. Secord

S. W. Sturdy

A. C. Warwick

R. C. Olby

J. A. Secord

H. S. Torrens

A. C. Warwick

Gill Hudson

C. J. Lawrence

J. A. Secord

A. C. Warwick

Gill Hudson

D. G. King-Hele

C. J. Lawrence

Dorinda Outram 
Council, 1991-92

P. J. Bowler

Janet Browne

Elspeth Crawford

J. Harwood

Gill Hudson

Council, 1992-93

P. J. Bowler

W. F. Bynum

Elspeth Crawford

Patricia Fara

J. Harwood

Council, 1993-94

W. F. Bynum

Elspeth Crawford

P. R. Ellis

Patricia Fara

J. Harwood

Council, 1994-95

J. E. Agar

W. F. Bynum

P. R. Ellis

Patricia Fara

Sally M. Horrocks

Council, 1995-96

J. E. Agar

P. R. Ellis

G. J. N. Gooday

Penelope Gouk

Sally M. Horrocks

Council, 1996-97

J. E. Agar

P. R. Ellis

G. J. N. Gooday

Penelope Gouk
D. G. King-Hele

C. J. Lawrence

Dorinda Outram

Gerrylyn K. Roberts

D. G. King-Hele

Dorinda Outram

Gerrylyn K. Roberts

J. R. Topham

Sally M. Horrocks

Gerrylyn K. Roberts

Anne Secord

J. R. Topham

M. J. Kemp

A. Q. Morton

Anne Secord

J. R. Topham

M. J. Kemp

A. Q. Morton

J. S. Rowlinson

Anne Secord

M. J. Kemp

A. Q. Morton

J. S. Rowlinson 
Honorary members

George Sarton, 1948

D'Arcy Wentworth Thompson, 1948

Charles Sherrington, 1948

Charles Singer, 1956

Singer Prize winners

M. Ridley, 1980

S. Schaffer and Mari Williams, 1982

Not awarded, 1984

M. Shortland, 1986
Eva G. R. Taylor, 1961

H. Hamshaw Thomas, 1961

Alexandre Koyré, 1963

J. R. Partington, 1963

G. Gooday and M. Ben-Chaim, 1988

J. Topham and M. Harrison, 1990

J. Agar, 1992

D. Wright and P. Lucier, 1994 